Journal of Nonlinear Optical Physics \& Materials

Vol. 16, No. 4 (2007) 551-556

(C) World Scientific Publishing Company

\title{
AUTHOR INDEX VOLUME 16
}

Åagren $\mathrm{H}$, see Baev A

16 (2007) 157

Abu Hassan H, see Chuah LS

16 (2007) 497

Aimez V, see Suntsov $S$

Alberucci A \& Assanto G, Dissipative Self-Confined Optical Beams in

16 (2007) 401

Doped Nematic Liquid Crystals

Anbarasan PM, see Kumaresan P

16 (2007) 295

16 (2007) 255

Arecchi FT, see Residori $S$

16 (2007) 343

Arès R, see Suntsov $S$

Assanto G \& Peccianti M, Routing Light at Will

16 (2007) 401

16 (2007) 37

Assanto G, see Alberucci A

16 (2007) 295

Baert K, Libaers W, Kolaric B, Vallée RAL, van der Auweraer M, Clays K, Grandjean D, Di Vece M \& Lievens P, Development of Magnetic Materials for Photonic Applications

16 (2007) 281

Baert K, Wostyn K, Vallée RAL \& Clays K, Second-Order Nonlinear Optical Properties of Chromophore-Coated Particles: Symmetry Considerations

16 (2007) 27

Baev A, Welinder P, Erlandsson R, Henriksson J, Norman P \& Åagren H, A Quantum Mechanical - Electrodynamical Approach to Nonlinear Properties: Application to Optical Power Limiting with Platinum-Organic Compounds

Bai B, see Canfield BK

Bezryadina A, see Suntsov $S$

Bhadra S, see Roy $S$

Blinov LM, Cipparrone G, Lazarev VV, Pagliusi P, Rugiero T \& Umanskii BA, Polarized Spectra of Amplified Spontaneous Emission and Gain for Glycerin Solutions of Dye Rhodamine-640

Blinov LM, Lazarev VV, Palto SP, Cipparrone G, Mazzulla A \& Pagliusi P, Electric Field Tuning a Spectrum of Nematic Liquid Crystal Lasing with the Use of a Periodic Shadow Mask

Bortolozzo U, Residori S \& Huignard J-P, Nonlinear Optical Applications of Photorefractive Liquid Crystal Light-Valves

Bortolozzo U, see Residori $S$

16 (2007) 157

16 (2007) 317

16 (2007) 401

16 (2007) 119

16 (2007) 519

Bunning TJ, see Serak SV

Canfield BK, Kujala S, Husu H, Kauranen M, Bai B, Laukkanen J, Kuittinen M, Svirko Y \& Turunen J, Local-Field and Multipolar Effects in the Second-Harmonic Response of Arrays of Metal Nanoparticles 
Cheng $\mathrm{N}$, see He GS

16 (2007) 137

Chrastina D, see Trita A

Christodoulides DN, see Suntsov $S$

Chuah LS, Hassan Z \& Abu Hassan H, The Study of Pd Schottky

16 (2007) 207

16 (2007) 401

Contact on Porous GaN for UV Metal-Semiconductor-Metal

(MSM) Photodetectors

Cipparrone G, see Blinov LM

Cipparrone G, see Blinov LM

Clays K, see Baert $K$

Clays K, see Baert K

Collings N, see Trushkevych $O$

Cook G, see Evans DR

Cristiani I, see Trita A

Crossland WA, see Trushkevych $O$

de Luca A, see Pezzi L

Degiorgio V, see Trita A

Derose C, see Norwood RA

Dhinaa AN, see Nooraldeen $A Y$

Di Vece M, see Baert $K$

Diaz A, Park JH \& Khoo IC, Design and Transmission-Reflection Properties of Liquid Crystalline Optical Metamaterials with Large Birefringence and Sub-Unity or Negative-Refractive Index

Diaz A, see Khoo IC

Ding YJ, see $M u X$

Döbeli M, see Trita A

Donmez E, see Karakas $A$

Dou N, see Li C

Elmali A, see Karakas $A$

Elmali A, see Karakas $A$

Elmali A, see Karakas $A$

Elmali A, see Karakas $A$

Enami Y, see Norwood $R A$

Erlandsson R, see Baev A

Evans DR \& Cook G, Bragg-Matched Photorefractive Two-Beam Coupling in Organic-Inorganic Hybrids

Fallahi M, see Norwood RA

Gan $\mathrm{H}$, see Norwood $R A$

Gao S \& He S, Four-Wave Mixing in Left-Handed Materials

Georgiou A, see Trushkevych $O$

Grandjean D, see Baert $K$

Greenlee C, see Norwood RA

Hassan Z, see Chuah LS

He GS, Zheng Q, Cheng N, Xu F \& Prasad PN, Optical

Phase-Conjugation Property of Three-Photon Excited Backward Stimulated Emission

He S, see Gao $S$

Henriksson J, see Baev A

Himmelhuber $\mathrm{R}$, see Norwood RA

16 (2007) 497

16 (2007) 75

16 (2007) 519

16 (2007) 27

16 (2007) 281

16 (2007) 307

16 (2007) 271

16 (2007) 207

16 (2007) 307

16 (2007) 199

16 (2007) 207

16 (2007) 217

16 (2007) 359

16 (2007) 281

16 (2007) 533

16 (2007) 381

16 (2007) 427

16 (2007) 207

16 (2007) 329

16 (2007) 101

16 (2007) 91

16 (2007) 247

16 (2007) 329

16 (2007) 505

16 (2007) 217

16 (2007) 157

16 (2007) 271

16 (2007) 217

16 (2007) 217

16 (2007) 485

16 (2007) 307

16 (2007) 281

16 (2007) 217

16 (2007) 497

Huignard J-P, see Bortolozzo $U$

16 (2007) 137

16 (2007) 485

16 (2007) 157

16 (2007) 217

16 (2007) 231

Huignard J-P, see Residori $S$

16 (2007) 343 
Husu H, see Canfield BK

16 (2007) 317

Iwanow R, see Suntsov $S$

16 (2007) 401

Kara H, see Karakas A

16 (2007) 329

Kara $\mathrm{H}$, see Karakas $A$

Karakas A \& Elmali A, Theoretical Studies on Linear and Nonlinear

16 (2007) 505

Optical Characterization and Optical Limiting Property of Pb(II), In(III) Chloride, Ni(II) Metallated

1,4,8,11,15,18,22,25-Octaalkylphthalocyanines

16 (2007) 247

Karakas A, Donmez E, Kara H \& Elmali A, Synthesis, Crystal Structure, Spectroscopic Studies and Ab-Initio Calculations on Third-Order Optical Nonlinearity of a Five-coordinate Chloroiron(III) Complex

16 (2007) 329

Karakas A, Elmali A, Yahsi Y \& Kara H, Third-Order Nonlinear Optic and Optical Limiting Properties of a MN(III) Transition Metal Complex

Karakas A, Unver H \& Elmali A, The Investigation of Electronic Properties and Microscopic Second-Order Nonlinear Optical Behavior of 1-Salicylidene-3-Thio-Semicarbazone

Kauranen M, see Canfield BK

Khoo IC, Diaz A, Kwon D, Werner DH, Liou J, Stinger M, Park JH, Kubo S \& Mallouk T, Nonlinear and Electro-Optics of Nano-Dispersed Nematic Liquid Crystals with Tunable Negative-, Zero-, and Positive Indices

Khoo IC, see Diaz A

Kim RH, see Lee $K$-S

Kivshar YS, see Neshev DN

Kolaric B, see Baert $K$

Krolikowski W, see Neshev DN

Kropachev O, see Norwood RA

Kubo S, see Khoo IC

Kuittinen M, see Canfield BK

Kujala S, see Canfield BK

Kumaresan P, Moorthy Babu S \& Anbarasan PM, Thermal, Dielectric Studies on Pure and Amino Acid (L-Glutamic Acid, L-Histidine, L-Valine) Doped Potassium Dihydrogen Phosphate Single Crystals Kwon D, see Khoo IC

Laukkanen J, see Canfield BK

Lazarev VV, see Blinov LM

Lazarev VV, see Blinov LM

Lee K-S, Kim RH, Prabhakaran P, Yang D-Y, Lim TW \& Park SH, Two-Photon Stereolithography

Li C, Xu G, Dou N \& Wang F, Low-Power All-Optical Switching Using a Fiber Sagnac Interferometer with a Pumped Nonlinear Coupler

Li P, see Shi K

Libaers W, see Baert $K$

Lievens P, see Baert $K$

Lim TW, see Lee $K$-S

Liou J, see Khoo IC

Liu D, see Xu $M$

16 (2007) 381

16 (2007) 533

16 (2007) 59

16 (2007) 1

16 (2007) 281

16 (2007) 1

16 (2007) 217

16 (2007) 381

16 (2007) 317

16 (2007) 317

16 (2007) 255

16 (2007) 381

16 (2007) 317

16 (2007) 75

16 (2007) 519

16 (2007) 59

16 (2007) 101

16 (2007) 457

16 (2007) 281

16 (2007) 281

16 (2007) 59

16 (2007) 381

16 (2007) 269 
Liu Z, see Shi K

16 (2007) 457

Loychik C, see Norwood $R A$

Lu M \& Chen X, Multiple Quasi-Phase Matching in Engineered

16 (2007) 217

Domain-Inverted Optical Superlattice

Luther-Davies B, see Zha $C$

Ma S, see $X u M$

Madden S, see Zha $C$

Makris KG, see Suntsov $S$

Makwani D \& Vijaya R, Frequency-Dependent Hyperpolarizability of Benzene Derivatives: Ab-Initio Calculations

Mallouk T, see Khoo IC

Mathine D, see Norwood RA

Mazzulla A, see Blinov LM

Merzylak Y, see Norwood RA

Milne WI, see Trushkevych $O$

Min Y, see Suntsov $S$

Montina A, see Residori $S$

Moorthy Babu S, see Kumaresan P

Morandotti R, see Suntsov $S$

$\mathrm{Mu}$ X, Ding YJ \& Zotova IB, From Nonresonant to

Resonance-Enhanced THz Generation

Neshev DN, Sukhorukov AA, Krolikowski W \& Kivshar YS, Nonlinear Optics and Light Localization in Periodic Photonic Lattices

Nolan DA, see Sysoliatin AA

Nooraldeen AY, Dhinaa AN \& Palanisamy PK, Nonlinear Optical Properties of Acid Orange 10 Dye by z-Scan Technique Using Ar ${ }^{+}$ Laser

16 (2007) 185

16 (2007) 49

16 (2007) 269

16 (2007) 49

16 (2007) 401

16 (2007) 367

16 (2007) 381

16 (2007) 217

16 (2007) 75

16 (2007) 217

16 (2007) 307

16 (2007) 401

16 (2007) 343

16 (2007) 255

16 (2007) 401

16 (2007) 427

$16(2007) 1$

16 (2007) 171

Norman P, see Baev A

Norwood RA, Derose C, Enami Y, Gan H, Greenlee C, Himmelhuber

16 (2007) 157 R, Kropachev O, Loychik C, Mathine D, Merzylak Y, Fallahi M \& Peyghambarian N, Hybrid Sol-Gel Electro-Optic Polymer Modulators: Beating the Drive Voltage/Loss Tradeoff

16 (2007) 217

Pagliusi P, see Blinov LM

Pagliusi P, see Blinov LM

16 (2007) 75

16 (2007) 519

16 (2007) 359

Palanisamy PK, see Nooraldeen $A Y$

16 (2007) 75

Palto SP, see Blinov LM

16 (2007) 533

Park JH, see Diaz A

16 (2007) 381

Park JH, see Khoo IC

16 (2007) 59

Park SH, see Lee $K-S$

Peccianti M, see Assanto $G$

Peyghambarian $\mathrm{N}$, see Norwood $R A$

Pezzi L, Veltri A, de Luca A \& Umeton C, Model for Molecular

16 (2007) 37

16 (2007) 217

Director Configuration in a Liquid Crystal Cell with Multiple Interfaces

16 (2007) 199

Prabhakaran P, see Lee $K-S$

16 (2007) 59

Prasad A, see Zha $C$

16 (2007) 49

Prasad PN, see He GS

16 (2007) 137 
Residori S, Bortolozzo U, Montina A, Arecchi FT \& Huignard J-P, A Novel Type of Optical Oscillator Based on a Liquid Crystal Gain Medium

16 (2007) 343

Residori S, see Bortolozzo $U$

Roy S \& Bhadra S, Study of Pulse Evolution and Optical Bistability

16 (2007) 231

Under the Influence of Cubic-Quintic Nonlinearity and Third Order Dispersion

Rugiero T, see Blinov LM

Saeung $\mathrm{P}$, see Yupapin PP

Salamo G, see Suntsov $S$

Schiek R, see Suntsov $S$

Serak SV, Tabiryan NV \& Bunning TJ, Nonlinear Transmission of Photosensitive Cholesteric Liquid Crystals Due to Spectral

Bandwidth Auto-Tuning or Restoration

Shi K, Li P \& Liu Z, Supercontinuum CARS Tweezers

Siviloglou GA, see Suntsov $S$

Smith A, see Zha $C$

Sohler W, see Suntsov $S$

Sorel M, see Suntsov $S$

Stegeman GI, see Suntsov $S$

Stinger M, see Khoo IC

Sukhorukov AA, see Neshev DN

Suntsov S, Makris KG, Siviloglou GA, Iwanow R, Schiek R,

Christodoulides DN, Stegeman GI, Morandotti R, Yang H, Salamo G, Volatier M, Aimez V, Arès R, Sorel M, Min Y, Sohler W, Wang $\mathrm{X}$, Bezryadina A \& Chen Z, Observation of One- and

Two-Dimensional Discrete Surface Spatial Solitons

Suwancharoen W, see Yupapin PP

Svirko Y, see Canfield BK

Sysoliatin AA \& Nolan DA, Optical Signal Processing in Dispersion Varying Fiber

Tabiryan NV, see Serak SV

Trita A, Cristiani I, Degiorgio V, Döbeli M, Chrastina D \& Von Känel H, Measurement of the Lifetime of Photo-Generated Free Carriers in SiGe Waveguides

16 (2007) 119

16 (2007) 519

16 (2007) 111

16 (2007) 401

16 (2007) 401

16 (2007) 471

16 (2007) 457

16 (2007) 401

16 (2007) 49

16 (2007) 401

16 (2007) 401

16 (2007) 401

16 (2007) 381

16 (2007) 1

16 (2007) 401

16 (2007) 111

16 (2007) 317

16 (2007) 171

16 (2007) 471

16 (2007) 207

Trushkevych O, Collings N, Crossland WA, Wilkinson TD, Georgiou A \& Milne WI, Projection of Holograms from Photorefractive Oaslms

16 (2007) 307

Turunen J, see Canfield BK

Umanskii BA, see Blinov LM

Umeton C, see Pezzi $L$

Unver $\mathrm{H}$, see Karakas $A$

Vallée RAL, see Baert $K$

Vallée RAL, see Baert $K$

van der Auweraer M, see Baert $K$

Veltri A, see Pezzi L

Vijaya R, see Makwani D

Volatier M, see Suntsov $S$

16 (2007) 317

16 (2007) 519

16 (2007) 199

16 (2007) 91

16 (2007) 27

16 (2007) 281

16 (2007) 281

16 (2007) 199

16 (2007) 367

16 (2007) 401

16 (2007) 207

Von Känel H, see Trita $A$

16 (2007) 101

Wang F, see Li C 
Wang $\mathrm{R}$, see Zha $C$

16 (2007) 49

Wang W, see $X u M$

16 (2007) 269

Wang X, see Suntsov $S$

16 (2007) 401

Welinder P, see Baev A

16 (2007) 157

Werner DH, see Khoo IC

16 (2007) 381

Wilkinson TD, see Trushkevych $O$

16 (2007) 307

Wostyn K, see Baert $K$

16 (2007) 27

Xia Y, see $X u M$

16 (2007) 269

$\mathrm{Xu} \mathrm{F}$, see He GS

16 (2007) 137

$\mathrm{Xu} \mathrm{G}$, see $\operatorname{Li} C$

16 (2007) 101

Xu M, Wang W, Ma S, Xia Y \& Liu D, Dynamical Addressing Optical Interconnection Based on One-Dimensional Nonlinear Photonic Crystal

Yahsi Y, see Karakas A

16 (2007) 269

16 (2007) 505

Yang D-Y, see Lee $K-S$

16 (2007) 59

Yang H, see Suntsov $S$

Yupapin PP, Saeung P \& Suwancharoen W, Coupler-Loss and

16 (2007) 401 Coupling-Coefficient Dependence of Bistability and Instability in a Fiber Ring Resonator: Nonlinear Behaviors

$16(2007) 111$

Zha C, Smith A, Prasad A, Wang R, Madden S \& Luther-Davies B, Properties and Structure of Ag-Doped $\mathrm{As}_{2} \mathrm{Se}_{3}$ Glasses

16 (2007) 49

Zheng Q, see He GS

16 (2007) 137

Zotova IB, see $M u X$

16 (2007) 427 